\title{
Tuberculosis: an Old but Still Fascinating Disease
}

\author{
C. Ruef
}

In this issue of Infection we are publishing five papers that deal with various aspects of tuberculosis [1-5]. The first two manuscripts are reviews on the topic of latent tuberculosis and its pathogenesis. While Cardona proposes that constant endogenous reinfection with Mycobacterium tuberculosis occurs [1], Ehlers discusses other hypotheses to explain latency, such as persistence of the bacteria in a "lazy" state within granulomatous lesions and an ongoing dynamic immunological interplay between $M$. tuberculosis and the host that requires continuous recruitment of cells into the granuloma [2]. He also discusses the view that dormant $M$. tuberculosis may reside within alveolar epithelial cells in the lung and in adipocytes, which may become reactivated by certain factors. I consider both views interesting to read and stimulating for further research.

On a more practical level, the manuscript by Lee et al. investigates the sensitivity, specificity and positive and negative predictive values of modern T-cell-based assays and the more traditional tuberculin skin test to diagnose latent tuberculosis infection in patients with end-stage renal disease on hemodialysis [3]. These papers are accompanied by a short report on interleukin- 6 blood levels in patients with tuberculosis. Correia et al. [4] found that IL-6 levels were increased in pulmonary tuberculosis independent of drug resistance. Finally, a case of spontaneous splenic rupture in the context of an immune reconstitution inflammatory syndrome is described in a HIV type 1-infected patient with tuberculosis [5]. While the splenic rupture was not primarily associated with tuberculosis, tuberculosis definitively played a role in the pathogenesis of the immune reconstitution inflammatory syndrome that occurred 10 days after initiating highly active anti-retroviral therapy. This case illustrates the complexity of treatment in patients co-infected with HIV and M. tuberculosis.

While five articles on tuberculosis in one issue of Infection may appear to be a lot, this aggregation of publications on one topic mainly illustrates the importance of tuberculosis and also the broad spectrum of issues that still requires ongoing research to better understand the pathogenesis, diagnosis and treatment of this infection, which still affects millions of people annually worldwide.

\section{References}

1. Cardona PJ: A dynamic reinfection hypothesis of latent tuberculosis infection. Infection 2009; 37: 80-86.

2. Ehlers S: Lazy, dynamic or minimally recrudescent? On the elusive nature and location of the Mycobacterium responsible for latent tuberculosis. Infection 2009; 37: 87-95.

3. Lee SSJ, Chou KJ, Su IJ, Chen YS, Fang HC, Huang TS, Tsai HC, Wann SR, Lin HH, Liu YC: High prevalence of latent tuberculosis infection in patients in end-stage renal disease on hemodialysis: Comparison of QuantiFERON-TB GOLD, ELISPOT, and tuberculin skin test. Infection 2009; 37: 96-102.

4. Correia JW, Freitas MV, Queiroz JA, PereiraPerrin M, Cavadas B: Interleukin- 6 blood levels in sensitive and multiresistant tuberculosis. Infection 2009; 37: 138-141.

5. Weber E, Günthard H, Schertler T, Seebach JD: Spontaneous splenic rupture as manifestation of the immune reconstitution inflammatory syndrome in an HIV type 1 infected patient with tuberculosis. Infection 2009; 37: 163-165.
Infection 2009; 37: 79

DOI 10.1007/s15010-009-3209-8

Published online: March 23, 2009 